\title{
Aleukemic Monocytic Leukemia Cutis
}

National Cancer Institute

\section{Source}

National Cancer Institute. Aleukemic Monocytic Leukemia Cutis. NCI Thesaurus. Code C5630.

Aleukemic leukemia cutis where the skin in infiltrated by neoplastic monocytes. 